\title{
Characteristics of patients with blastemal-type Wilms' tumour
}

\author{
Weronika Hajzler ${ }^{1}$, Joanna Kopera², Klaudia Kosek², Dorota Mazur², Iwona Rurańska³, \\ Tomasz Szczepański ${ }^{3}$, Aneta Pobudejska-Pieniążek ${ }^{3}$ \\ 'Doctoral School, Department of Pediatric Hematology and Oncology, Faculty of Medical Sciences in Zabrze, Medical \\ University of Silesia in Katowice, Poland \\ ${ }^{2}$ Students' Scientific Association, Department of Pediatric Hematology and Oncology, Faculty of Medical Sciences in Zabrze, \\ Medical University of Silesia in Katowice, Poland \\ ${ }^{3}$ Department of Pediatric Hematology and Oncology, Faculty of Medical Sciences in Zabrze, Medical University of Silesia in \\ Katowice, Poland
}

\section{ABSTRACT}

Wilms' tumour (nephroblastoma) is the most common malignant tumour of kidney in children and one of the 2 most common solid tumours located outside of the central nervous system. Nephroblastoma has a very good prognosis, but some histological types like blastemal-type require qualification to a high-risk group and intensification of treatment. We present the cases of 3 children diagnosed with nephroblastoma, blastemal-type. The first girl's treatment was unsuccessful, as there were 4 cancer recurrences. The second girl had primarily metachronous form of nephroblastoma, but during recurrence the blastemal-type was diagnosed. Despite intensive treatment, it ended with failure. The third girl had a tumour in the left kidney, which was transformed from the bilateral nephroblastomatosis. She remains in remission; the therapy ended on February 2018.

\section{KEY WORDS:}

Wilms' tumor, nephroblastoma, blastemal-type.

\section{INTRODUCTION}

Renal tumours comprise 6-8\% of childhood cancers, with nephroblastoma (Wilms' tumour) being the most common. It usually affects children between 2 and 5 years old, equally boys and girls. It develops from the remains of embryonic nephrogenic tissue, usually due to the damage of suppressor genes, which are responsible for regulation of cell growth, proliferation, and differentiation [1]. Currently survival from nephroblastoma exceeds $90 \%$ at 5 years due to the increased understanding of the biology of this tumour and improved treatment protocols [2]. There are 2 main Wilms' tumour treatment strategies: the SIOP (International Society of Paediatric Oncology) protocol used in Europe, and the NWTS/COG (National Wilms' Tumour Study/ Children's Oncology Group) protocol from the United States of America. The essential difference between the protocols is the use of preoperative chemotherapy in the SIOP protocol, compared to upfront surgery in the NWTS protocol [3]. The beneficial effects of preoperative chemotherapy are reduction of tumour size and prevention of intraoperative spillage of the tumour [3]. In our article we will mainly focus on the SIOP protocol because in the presented cases this type of protocol was used in the treatment. According to the SIOP protocol the clinical stage of Wilms' tumour is determined after preoperative chemotherapy and subsequent nephrectomy. The clinical classification has 5 stages: I-III) clinically localized disease, IV) hematogenous metastases

\section{ADDRESS FOR CORRESPONDENCE:}

Aneta Pobudejska-Pieniążek, Department of Pediatric Hematology and Oncology, Faculty of Medical Sciences in Zabrze, Medical University of Silesia in Katowice, Poland, e-mail: apobudejska@interia.pl 
or lymph node metastases outside the abdominopelvic region, and V) bilateral Wilms tumour [4].

Microscopic structure of nephroblastoma is associated with prognosis, and it us allows to distinguish 3 groups of tumours according to their malignancy: 1) low-risk group, which includes cystic partially differentiated and completely necrotic nephroblastoma; 2) intermediate-risk group - epithelial, stromal, mixed, regressive types and nephroblastoma with focal anaplasia; and 3) high-risk group - blastemal type, nephroblastoma with diffuse anaplasia [4].

The following article describes various courses of disease in 3 cases diagnosed primarily or during recurrence as blastemal-type Wilms' tumours.

\section{PATIENT CASE STUDY NO. 1}

A 5-year-old girl was admitted to the Paediatric Haematology and Oncology Department of Clinical Hospital No. 1 in Zabrze with the suspicion of Wilms' tumour. The girl's parents had noticed the elevation and asymmetry of the abdominal wall and reported their concerns to their general practitioner, who ordered an ambulatory ultrasound test of the abdominal cavity, which revealed the presence of an oval tumour about $10 \mathrm{~cm}$ in diameter in the left upper quadrant. The girl was immediately transferred with an initial diagnosis of Wilms' tumour to our department. On admission there were no other symptoms suggesting disease. Abdominal and pelvic CT showed a tumour of size $11.5 \mathrm{~cm} \times 10 \mathrm{~cm} \times 9 \mathrm{~cm}$ with no radiological signs of infiltration of neighbouring structures. Lung metastases were ruled out on imaging studies. A 4-week preoperative chemotherapy (vincristine, actinomycin D) was initiated according to the SIOP 2001/2006 protocol, then left-sided nephrectomy was performed; resection of the tumour was radical. The histopathological examination showed nephroblastoma, blastemal-type - a high-risk tumour. The case was categorized as clinical stage I. 27-week postoperative chemotherapy was performed with the use of actinomycin D, vincristine, and doxorubicin. Due to the drug-induced polyneuropathy: gait disturbance, bilateral eyelid drooping, double vision, the dose of vincristine was reduced to $2 / 3$. As a result neurological symptoms were significantly reduced. In addition, chemotherapy was complicated by renal tubular acidosis. Post-treatment imaging tests confirmed remission of the primary disease. After 2 months, a local recurrence was diagnosed - the control CT test of the abdominal cavity revealed a hypodense mass lesion measuring up to $1.4 \mathrm{~cm} \times 1.6 \mathrm{~cm}$ in the paraaortic region, below the left renal artery stump. During the next procedure the tumour was removed, and a histopathological examination confirmed the previous diagnosis. The surgery was additionally complicated by the obstruction of the gastrointestinal tract, which required reoperation during which the intestine was released from the adhe- sions. Second-line chemotherapy was introduced: $2 \mathrm{cy}$ cles of CCE (cisplatin, cyclophosphamide, etoposide) and 2 cycles of ICE (ifosfamide, carboplatin, etoposide) according to the SIOP 2001/2006 protocol, followed by abdominal and pelvic radiotherapy. The child underwent a haematopoietic cell mobilization procedure to secure the material for possible autograft. Then, the autotransplantation had to be postponed due to severe long-lasting bone marrow aplasia and Fanconi syndrome, both caused by the current chemotherapy. The second recurrence was detected after 6 months. The abdominal CT scan revealed a tumour measuring $4.6 \mathrm{~cm} \times 2.1 \mathrm{~cm} \times 3.8 \mathrm{~cm}$ located in the retroperitoneal space near the tumour bed and closely abutting the spleen. The tumour and the spleen's inferior pole were removed; radicality of the resection was doubtful. The initiated treatment included preoperative chemotherapy for disseminated disease, according to the SIOP 2001/2006 protocol. Autologous stem cell transplantation was planned. After 2 weeks, due to the toxicity of the chemotherapy (prolonged aplasia, worsening of renal tubular acidosis) the treatment was discontinued. After stabilization of the girl's clinical condition, an autologous stem-cell transplantation procedure was performed. The autograft was complicated by severe side effects and veno-occlusive disease (VOD). After 4 months, the third recurrence occurred. MRI of the abdominal cavity showed a solid mass lesion measuring $5.5 \mathrm{~cm} \times 4.5 \mathrm{~cm}$ $\times 3 \mathrm{~cm}$ in Douglas' pouch, which was removed surgically. Another attempt was made on chemotherapy with irinotecan and dacarbazine and irradiation of the abdominal cavity and pelvis, with a dose limitation due to previous radiotherapy. Two months after the surgery, a fourth recurrence was detected - a tumour in the area of the liver. The girl was qualified for palliative treatment. She died 3 years after the first diagnosis. Apart from the last recurrence, all the previous relapses were confirmed to be Wilms' tumours by histopathological examination.

\section{PATIENT CASE STUDY NO. 2}

A 9-year-old girl was admitted to the Paediatric Haematology and Oncology Department of Clinical Hospital No. 1 in Zabrze due to recurrence of Wilms' tumour. She was previously treated in another hospital in 2002 due to a right kidney nephroblastoma, stage IV (with lung metastases), an intermediate-risk group; after treatment consisting of chemotherapy and surgery the patient was in remission. Five years after the end of the initial treatment the parents were concerned about an increased circumference of girl's abdomen, and she was admitted to local Paediatric Surgery Department. Suspicion of Wilms' tumour recurrence was made by a paediatric surgeon by ultrasound test and the girl was immediately referred to our department. On admission the child manifested the following symptoms: constipation, abdominal pain, and protuberant abdomen. The abdominal and pelvic CT 
test showed a solid mass of the left kidney (size of $16 \mathrm{~cm}$ $\times 11 \mathrm{~cm} \times 10 \mathrm{~cm}$ ), which was compressing the spleen and pancreas and modelling the intestine. Lung metastases was ruled out in imaging studies. Preoperative chemotherapy was initiated according to the SIOP 2001/2006 protocol. The treatment was additionally complicated by acute cholecystitis, pancreatitis, and Horner syndrome. Kidney-sparing surgery was postponed due to unsatisfactory tumour mass reduction after 4 weeks. Therefore, chemotherapy with vincristine and actinomycin $\mathrm{D}$ was continued, the dose of vincristine was reduced to $2 / 3$ due to the intensification of drug-induced polyneuropathy (steppage gait, decreased muscular force in the lower limbs). The control imaging tests showed no significant improvement. The second-line chemotherapy was initiated according to the SIOP 2001/2006 protocol: 2 cycles of CCE (cisplatin, cyclophosphamide, etoposide). The tumour size was reduced to $4.6 \mathrm{~cm} \times 2.3 \mathrm{~cm} \times 8.3 \mathrm{~cm}$. After the chemotherapy the tumour was resected, unfortunately incompletely. After the procedure a histopathological examination confirmed the recurrence of nephroblastoma, the type with stroma dominance - intermediate risk. The patient underwent irradiation on the tumour bed. Then postoperative chemotherapy was applied as a treatment for clinical stage III, intermediate-risk group (vincristine, actinomycin D, doxorubicin). Six months after the surgery, the imaging tests showed up a solid mass in the pelvis, measuring up to $4.0 \mathrm{~cm} \times 3.9 \mathrm{~cm}$ $\times 4.6 \mathrm{~cm}$, modelling the urinary tract. Second-line therapy was introduced: pre-operative 2 cycles of ICE (ifosfamide, carboplatin, etoposide) and another 2 cycles after the surgery. The histopathological examination revealed a blastemal-type nephroblastoma. Four months later, imaging tests showed progression of the disease. The abdominal and pelvic MRI showed the presence of $3 \mathrm{tu}-$ mours: the first one in the left kidney measuring $6 \mathrm{~cm}$ $\times 7.2 \mathrm{~cm}$, infiltrating the ureter, the second one between the spleen and superior pole of the left kidney measuring $7.2 \times 5.3 \mathrm{~cm}$, and the third tumour located under the spleen measuring $8 \mathrm{~cm} \times 5.6 \mathrm{~cm}$. Also several enlarged lymph nodes were observed. ICE chemotherapy (ifosfamide, carboplatin, and etoposide) was continued, additionally combined with topotecan. Symptoms of encephalopathy appeared after the first dose of topotecan. Brain scans showed no abnormalities. The girl was transferred to a different oncologic hospital, for further surgical treatment on the parents' request. The child died before the planned surgery, 1.5 years after the first Wilms' tumour recurrence.

\section{PATIENT CASE STUDY NO. 3}

An 18-month-old girl was transferred from a local hospital to the Paediatric Haematology and Oncology Department of Clinical Hospital No. 1 in Zabrze due to a left kidney tumour. The pathological structure was found during the abdominal ultrasound examination, performed because of gastroenteritis. There were no alarming symptoms indicated an existing tumour, and the physical examination did not reveal any abnormalities. Abdominal and pelvic CT tests performed in our department confirmed a pathological solid mass measuring up to $5 \mathrm{~cm} \times 4.9 \mathrm{~cm} \times 4.4 \mathrm{~cm}$ in the inferior pole of the left kidney, and the test also showed bilateral features of nephroblastomatosis, with a majority on the left side. Wilms' tumour of the left kidney was diagnosed on the basis of bilateral nephroblastomatosis. Genetic testing ruled out the mutation of the WT1 gene. Lung metastases was ruled out in imaging studies. Preoperative chemotherapy was initiated according to the SIOP 2001/2006 protocol, followed by left-sided nephrectomy; resection of the tumour was radical. After the procedure a histopathological examination revealed nephroblastoma, blastemal-type, high-risk group. In addition, a few nephroblastomatosis centres were found in the removed kidney. Twenty-seven-week postoperative chemotherapy was continued according to the treatment protocol for the clinical Stage I, high-risk group. After the chemotherapy the cycle control examinations confirmed the presence of remission of the disease. Then, treatment of the nephroblastomatosis was initiated according to the SIOP 2001/2006 protocol (vincristine and actinomycin D - every 4 weeks within the period of 12 months). Cardiologic consultation during chemotherapy showed left ventricular dilatation and minimal tricuspid regurgitation, which was not previously observed and required further control. Nevertheless, there were no significant complications of the treatment. Currently, the girl has remained in remission for 36 months since the end of treatment.

\section{DISCUSSION}

Increased intensity of postoperative treatment in blastemal-type of nephroblastoma in SIOP 2001/2006 protocol improved outcomes in comparison to SIOP 93-01 protocol: 5-year event-free survival (EFS) of $80 \%$ compared to $67 \%$ and overall survival (OS) of $88 \%$ compared to $67 \%$, but still this histological type of tumour has a worse prognosis than the other types [5]. Blastemal-type tumour consists of over $66 \%$ of blastemal cells in a tumour with more than $33 \%$ of cells viable [4]. Blastemal cells respond poorly to treatment, which is associated with greater risk of recurrence and lower EFS and OS rates [6-8]. In this article we presented 3 cases of patients diagnosed with blastemal-type Wilms' tumours. All histopathological examinations were confirmed by the referenced pathologist. WT1 gene mutation was ruled out only in one presented case, and there were no genetic examinations of tumour tissue. Only 1 girl remains in remission, in the other 2 cases the course of the treatment was complicated with recurrences, and intensive treatment 
gave many side effects and ended with failure. Data from Southern and Eastern Europe state that high-risk or unfavourable histology cases have 2.3 times higher relapse rate and 5.6 times higher respective death rate than low and intermediate risk groups or favourable histology cases [6].

In a Chinese retrospective study including patients treated according to the COG protocol the recurrence rate was $45.5 \%$ in blastemal-type Wilms' tumour, while EFS and OS were $54.5 \%$ and $68.2 \%$, respectively [7]. In the COG protocol there are only 2 histological types: with favourable histology and with unfavourable histology indicating presence of anaplasia [7]. Blastemal-type is classified as favourable histology and is not considered as a high-risk factor, in contrast to the SIOP protocol [7]. In a Japanese study the 5-year overall survival (OS) in blastemal-type was $65.4 \%$, in mixed type it was $90.1 \%$, in epithelial type it was $100 \%$, and in mesenchymal type it was 93.3\% [8]. Patients were treated according to the JWiTS (Japanese Wilms' Tumour Study) protocol based on the NWTS-5 regimen consisting of initial radical operation followed by adjuvant chemotherapy and radiotherapy [8]. In contrast, in another Japanese study that used the same JWiTS protocol in the treatment, there were no significant difference in 10-year relapse-free survival (RFS) and OS (78.8\% vs. $84.5 \%, p=0.201)$ between blastemal-type and non-blastemal Wilms' tumour [9]. Two of the presented studies from countries outside Europe suggest the necessity of blastemal-type extraction from the favourable histology group and modification of treatment in this group $[7,8]$.

SIOP 2001/2006 risk stratification uses clinical stage, histology, tumour volume, and responsiveness to initial chemotherapy [10]. A new protocol, UMBRELLA SIOP2016, validates new prognostic factors among, including blastemal tumour volume, and retrospective studies indicate that approximately 20-50 mL of residual blastemal volume could be used as a potential stratifying biomarker, but there is a need to further confirm these results $[4,11]$.

In one of the presented cases histological transformation of the was observed. The first histopathological examination revealed metachronous nephroblastoma, but after recurrence the blastemal-type Wilms' tumour was detected. Metachronous nephroblastoma is rare and occurs only in $2-3 \%$ of cases [12].

The nephroblastomatosis described in the third case increases the risk of Wilms' tumour development. Nephroblastomatosis is remaining embryonic renal tissue, which can be the base for carcinogenesis in 35\% of cases [13]. Nephroblastomatosis is also a risk factor of metachronous and bilateral form of Wilms' tumour $[4,14]$. In the SIOP protocol nephroblastomatosis has an individual protocol for treatment [4]. In the third presented case nephroblastoma developed on the basis of the nephroblastomatosis. The tumour was diagnosed accidentally during a USG examination, which shortened the potential time of disease development. Histopatholog- ical examination showed several centres of nephroblastomatosis in both kidneys, which can be qualified as small Wilms' tumours. It is possible that early diagnosis kept the patient away from the development of the bilateral form of nephroblastoma.

In the 2 presented cases we observed severe treatment-related side-effects. The most common were vincristine-related neurological complications: motor and sensory polyneuropathy in lower extremities, Horner syndrome, visual impairment, drooping eyelid, and encephalopathy. One girl had Fanconi syndrome, which is often caused by the use of ifosfamide [15]. In this case Fanconi syndrome had occurred already during first line of chemotherapy; however, it escalated after ifosfamide and cyclophosphamide application. Another rare complication observed was VOD after the haematopoietic stem cell autotransplantation procedure. In a Dutch study on the group of 511 patients with nephroblastoma, the VOD was present in $8 \%$ of cases [16]. Vincristine and actinomycin $\mathrm{D}$ use is known as a possible risk factor for VOD development [17]. Another risk factor of VOD is radiotherapy of the liver region [18], which was probably the cause of the VOD in the presented case. Moreover, one of the girls developed left ventricle dilation and minimal tricuspid valve regurgitation, after the use of anthracyclines.

\section{CONCLUSIONS}

Histological type of tumour has a significant impact on the prognosis in nephroblastoma. In 2 patients the course of the disease was tragic and treatment of the disease ended with failure. The 3 cases presented in this manuscript confirm previous observations that blastemal-type Wilms' tumour is associated with worse response to chemotherapy and high risk of treatment failure.

\section{DISCLOSURE}

The authors declare no conflict of interest.

\section{REFERENCES}

1. Marciniak P, Wachowiak J. Nerczak zarodkowy u dzieci i młodzieży czynniki prognostyczne w pierwszej manifestacji guza oraz we wznowie. Medycyna Wieku Rozwojowego 2009; 13: 59-65.

2. Phelps H, Kaviany S, Borinstein S, et al. Biological Drivers of Wilms Tumour Prognosis and Treatment. Children 2018; 5: 145.

3. Bhatnagar S. Management of Wilms' tumour: NWTS vs SIOP. J Indian Assoc Pediatr Surg. 2009; 1: 6-14.

4. Vujanić GM, Gessler M, Ooms AHAG, et al. The UMBRELLA SIOP-RTSG 2016 Wilms tumour pathology and molecular biology protocol. Nat Rev Urol 2018; 15: 693-701.

5. van del Heuvel-Eibrink MM, van Tinteren H, Bergeron C, et al. Outcome of localised blastemal-type Wilms tumour patients treated according to intensified treatment in the SIOP WT 2001 protocol, 
a report of the SIOP Renal Tumour Study Group (SIOP-RTSG). Eur J Cancer 2015; 51: 498-506.

6. Doganis D, Zborovskaya A, Trojanowski M, et al. Wilms tumour event-free and overall survival in Southern and Eastern Europe: Pooled analyses of clinical data from four childhood cancer registries (1999-2017). Eur J Cancer 2019; 115: 37-46.

7. Huang J, Zhang Y, Zhen Z, et al. The prognosis of prechemotherapy blastemal predominant histology subtype in Wilms tumour: A retrospective study in China. Pediatr Blood Cancer 2020; 67: 1-8.

8. Kinoshita $Y$, Suminoe A, Inada $\mathrm{H}$, et al. The prognostic significance of blastemal predominant histology in initially resected Wilms' tumours: A report from the Study Group for Pediatric Solid Tumours in the Kyushu Area, Japan. J Pediatr Surg 2012; 47: 2205-2209.

9. Koshinaga T, Takimoto T, Okita H, et al. Blastemal predominant type Wilms tumour in Japan: Japan Children's Cancer Group. Pediatr Int 2019; 61: 351-357.

10. Dome JS, Perlman EJ, Graf N. Risk Stratification for Wilms Tumour: Current Approach and Future Directions. Am Soc Clin Oncol Educ B 2014; 34: 215-223.

11. Graf N, van Tinteren H, Pritchard-Jones K, et al. Is absolute blastema volume after preoperative chemotherapy in nephroblastoma relevant for prognosis. Pediatr Blood Cancer 2011; 57: 741-742.

12. Mambié Meléndez M, Guibelalde Del Castillo M, Nieto Del Rincón N, et al. Metachronous bilateral Wilms' tumour. An Esp Pediatr 2002; 56: $247-250$.

13. Bruce G, Nzekwu E, Cook AJ, et al. Case report: Diffuse hyperplastic perilobar nephroblastomatosis complicated by a unilateral Wilms tumour: diagnosis, treatment and follow-up. BMC Research Notes 2018; 11: 396.

14. Bergeron C, Iliescu C, Thiesse P, et al. Does nephroblastomatosis influence the natural history and relapse rate in Wilms' tumour? A single centre experience over 11 years. Eur J Cancer 2001; 37: 385-391.

15. Burk CD, Restaino I, Kaplan BS, et al. Ifosfamide-induced renal tubular dysfunction and rickets in children with Wilms tumour. J Pediatr 1990; 117: 331-335.

16. Bisogno G, de Kraker J, Weirich A, et al. Venoocclusive disease of the liver in children treated for Wilms tumour. Med Pediatr Oncol Suppl 1997; 29: 245-251.

17. Ortega JA, Donaldson SS, Ivy SP, et al. Venoocclusive disease of the liver after chemotherapy with vincristine, actinomycin D, and cyclophosphamide for the treatment of rhabdomyosarcoma. A report of the Intergroup Rhabdomyosarcoma Study Group. Childrens Cancer Group, the Pediatric Oncology Group, and the Pediatric Intergroup Statistical Center. Cancer 1997; 79: 2435-2439.

18. Kim J, Jung Y. Radiation-induced liver disease: current understanding and future perspectives. Exp Mol Med 2017; 49: e359. 